\title{
Urologist-led smoking cessation: a way forward through implementation science
}

\author{
Richard S. Matulewicz ${ }^{1,2,3}$, Danil V. Makarov ${ }^{1,2,3}$, Scott E. Sherman ${ }^{2,3}$, Sarah A. Birken ${ }^{4}$, Marc A. Bjurlin ${ }^{5,6}$ \\ ${ }^{1}$ Department of Urology, NYU Grossman School of Medicine, New York, NY, USA; ${ }^{2}$ Department of Population Health, NYU Grossman School \\ of Medicine, New York, NY, USA; ${ }^{3}$ Manhattan Campus, VA NY Harbor Health System, New York, NY, USA; ${ }^{4}$ Wake Forest School of Medicine, \\ Winston-Salem, NC, USA; ${ }^{5}$ Lineberger Comprehensive Cancer Center, University of North Carolina, Chapel Hill, NC, USA; ${ }^{6}$ Department of \\ Urology, University of North Carolina, Chapel Hill, NC, USA \\ Correspondence to: Richard S. Matulewicz, MD, MS. 227 E 30 $0^{\text {th }}$ St., Room 629G, New York, NY 10017, USA. \\ Email: Richard.Matulewicz@nyulangone.org.
}

Submitted Aug 27, 2020. Accepted for publication Sep 11, 2020.

doi: $10.21037 /$ tau-20-1204

View this article at: http://dx.doi.org/10.21037/tau-20-1204

\section{Introduction}

Cigarette smoking is responsible for one in every five deaths and is the single greatest preventable cause of disease worldwide (1). Cigarette smoking is associated with several genitourinary disease processes, but is most causally linked with bladder cancer. Current smokers have a threefold increased risk of bladder cancer compared with nonsmokers and an almost twofold greater risk than among former smokers (2). Smokers also have an estimated $40 \%$ higher risk of developing renal cell carcinoma, higher rates of infertility and erectile dysfunction, and worse outcomes when treated for prostate cancer (3-9).

Considerable progress has been made at the populationlevel to help people either avoid or quit smoking. Several recent studies, including one by the United States Surgeon General, have reaffirmed the benefits and reinforced the need for more widespread delivery of smoking cessation treatment by frontline clinicians who manage smokingrelated diseases. Urologists have the most leverage in helping bladder cancer patients stop smoking but infrequently deliver guideline concordant smoking cessation treatment $(10,11)$. Therefore, we must understand the barriers to the routine provision of smoking cessation counseling by urologists.

It is time for urologists to leverage the momentum generated by public policy success and treatment advances to help patients quit smoking. In this commentary, we aim to review some recently published data on the relationship between smoking and bladder cancer outcomes then outline a path for the urologic community to provide routine smoking cessation counseling. It is our goal is to use implementation and dissemination science to help others begin the process of studying and "bridging the chasm" between evidence-based smoking cessation treatment strategies and urologists delivering this care.

\section{Smoking after the diagnosis of bladder cancer}

Smoking affects patients with bladder cancer far past their initial diagnosis. A recent prospective, multicenter cohort study evaluated the impact of smoking on pathological response to neoadjuvant chemotherapy in patients undergoing radical cystectomy for bladder cancer (12). The association of smoking status at the time of treatment with pathological outcomes demonstrated that former and current smokers were significantly less likely to achieve a complete pathological response [odds ratio (OR) 0.37 and 0.34 , respectively], while current smoking status was significantly associated with a greater likelihood of no pathological response (OR 2.5). This adds to data from several past systematic reviews as well as a recently published meta-analysis that have demonstrated the deleterious effect continued smoking has on nearly

\footnotetext{
$\wedge$ ORCID: 0000-0003-0757-0885.
} 
all aspects of bladder cancer treatment: surgery, systemic chemotherapy, and radiation (13-15). As we continue to investigate possible molecular drivers that may, in part, explain the poor pathological outcomes observed among smokers, clinicians need to do their part to assure that smoking cessation treatment is provided as part of comprehensive bladder cancer care.

\section{Where is the gap in providing smoking cessation care?}

The recent Surgeon General's report details the impressive progress made toward reducing tobacco use and the burden of tobacco-related disease through population-level policies and impactful treatment discoveries $(16,17)$. However, despite these improvements, over 30 million Americans continue to smoke. The majority of smokers want to quit but very few are able. Smokers with bladder cancer principally rely on their urologists for medical information and guidance-more than their primary care physicians $(11,18)$. Counseling from a urologist was cited as the leading motivator in cessation attempts (19) and smokers who were advised to quit by their urologist were more than four times more likely to quit than if they were not counseled (20). However, only one fifth of urologists offer any smoking cessation treatment to their patients with bladder cancer $(10,21)$.

The lack of smoking cessation treatment delivered by clinicians very likely has downstream consequences which contribute to high rates of continued smoking among cancer survivors. A recent study in $7 A M A$ demonstrated that the prevalence of adults who currently smoke is highest among tobacco-related cancer survivors (which include kidney and bladder cancer) and that these survivors had a significantly higher odds of continued smoking compared to survivors of non-tobacco related cancers (22). The persistence of smoking, even in the face of a tobaccorelated cancer diagnosis, represents a failed opportunity to effectively intervene with evidence-based smoking cessation treatment. Calls to action for urologic cancer providers to support, encourage, and counsel our patients to quit smoking are common (23) but a gap in care remains. In the last decade, only one study has been published that prospectively reported the implementation and effectiveness of a smoking cessation program in the urology clinic (20).

All patients diagnosed with bladder cancer will see their urologist at least once each year and each visit can be viewed as a teachable moment to encourage patients toward smoking cessation. In the last 10 years, there have been 93
Cochrane Systematic Reviews published that describe the evidence for various techniques and strategies for smoking cessation treatment, such as behavioral counseling or FDA-approved pharmacotherapy. Despite the availability of these evidence-based techniques and strategies, only $30 \%$ of adult smokers use evidence-based approaches to support their quit efforts (17). While the efficacy of these smoking cessation strategies and their intended settings vary, none of their purported benefits can be realized if the interventions are not employed in routine clinical practice. Physician recommendation is one of the single biggest predictors of attempts to quit and suboptimal delivery of effective treatments may contribute to low quit rates (11). Additionally, the slow passive diffusion of evidence-based interventions (EBIs) from research studies to clinical use is estimated to be roughly 17 years (24) and only about $50 \%$ of EBIs ever actually reach the clinic (25). This gap between research and practice limits the public health impact of expensive trials and reduces the return on investment of the public's research dollars.

\section{Bridging the gap between evidence and clinical practice}

The field of dissemination and implementation science is a means of studying, approaching, and promoting the uptake of EBIs into routine clinical use (26). It is a transdisciplinary research field that considers the multi-level (patient, provider, organization, policy) barriers and facilitators of EBIs and leverages theory-based approaches that overcome or leverage these determinants. As mentioned, passive diffusion (e.g., provider-to-provider recommendation) or even active dissemination (e.g., clinical guidelines) of EBIs is unlikely to change clinical behavior. Efforts to facilitate implementation are essential to promote guideline adherence in clinical practice and requires buy-in from diverse stakeholders, including primary care providers, specialists, and patients as well has health plans and health care systems. However, each stakeholder faces unique barriers to broader smoking cessation activities, mandating a flexible approach.

Integrating smoking cessation into comprehensive specialty care treatment can help patients in many ways, as these physicians are usually trusted, long-term providers of care. Urologic and cancer society practice guidelines emphasize the importance of using evidence-based smoking cessation treatments for smokers with cancer. However, most guidelines are not accompanied by any implementation tool to help actually deliver this care (27). 
Table 1 Patient and physician/hospital system smoking cessation resources

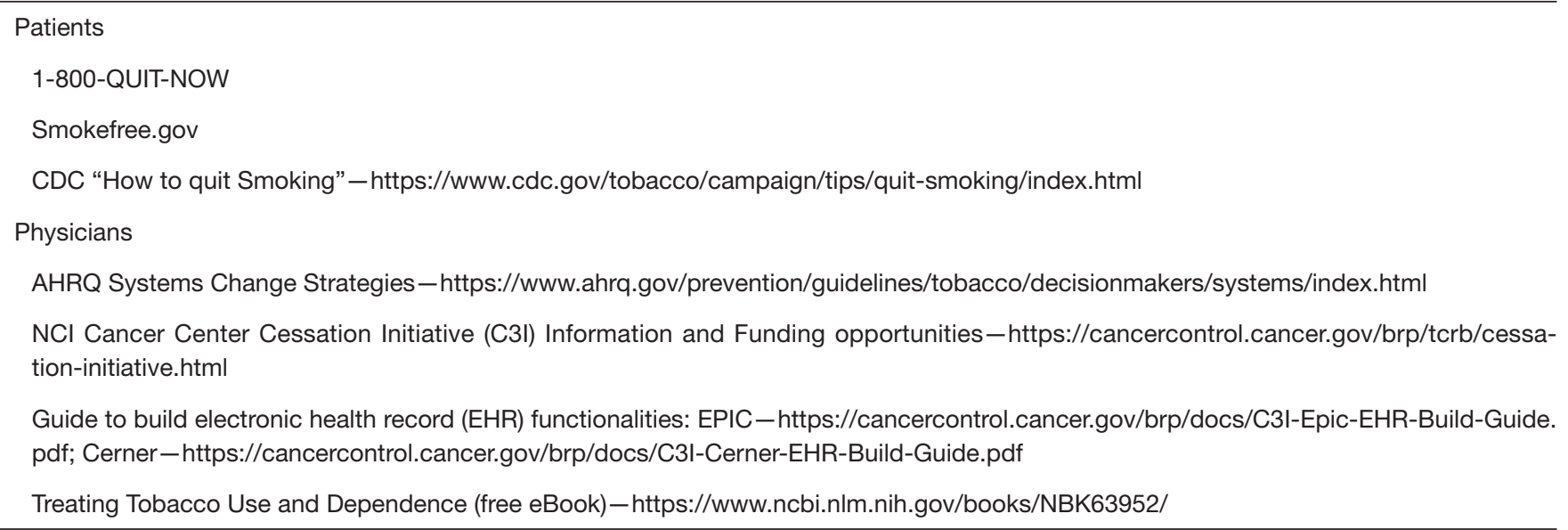

This is an issue because physicians frequently cite several barriers to providing smoking cessation care that are not considered by guideline recommendations (10). For example, despite the recommendation to engage in counseling, urologists often believe that this is outside the scope of the specialist and therefore infrequently offered (10). Lack of visit time and insufficient insurance reimbursement are frequently mentioned as another difficulty to providing preventive services such as smoking cessation treatment (28). Health systems that offer coverage of tobacco-dependence treatment result in increased selfreported sustained abstinence rates at relatively low costs since nearly all smoking cessation interventions are costeffective (29). Enabling physicians to deliver smoking cessation treatment requires an approach addresses these purported barriers. However, many of these barriers are not well studied, effectively limiting delivery.

Fortunately, well designed and validated process models and conceptual frameworks, such as the VA Quality Enhancement Research Initiative (QUERI), Consolidated Framework for Implementation Research (CFIR), and Theoretical Domains Framework (TDF) are available to help bridge the gap between evidence and clinical practice (30-32). These models are actionable and provide theorybased support for implementation and help provide a more standardized approach and understanding of implementation challenges. Using these frameworks also provides shared language and organization to grow the field and maximize transferrable knowledge. For example, the successful implementation of EBIs for smoking cessation care depends on factors at the level of the health care provider (e.g., knowledge, skills, outcome expectancies), the environment (e.g., availability of time, resources), and the patient (e.g., quit motivation). The National Cancer Institute (NCI) has reinforced the importance of using these strategies as essential research priorities with its Cancer Center Cessation Initiative (C3I) program (33). Notably, successful implementation depends on alignment among the EBI, the context into which it needs to be implemented, and the strategies used to implement it. Additional implementation science approaches, such as adaptation, can help to align EBIs, contexts, and implementation strategies, and scale efforts to diverse practice settings $(34,35)$.

\section{Looking forward}

As the field of implementation science grows and more urologists are trained in its methods, we will learn more about the determinants of smoking cessation care delivery. Opportunities through National Institutes of Health such as Training Institute for Dissemination and Implementation Research in Cancer (TIDIRC) can provide either formal or self-directed learning in these methods. Additionally, engaging in partnerships with implementation scientists, similar to biostatisticians or other content experts, during research design is essential to maximize the reach of findings. For an immediate start on implementing evidence-based smoking cessation strategies into daily practice, we have compiled some publically available resources that can help (Table 1).

The field of urology can and should be instrumental to smoking cessation progress at the physician-level. It will be essential to explore and better understand factors related to individual urologists' beliefs, behaviors and practices, health systems resources, clinic environments and support, 
as well as patient preferences. However, there are several fundamental implementation science questions that must first be answered: What is the true prevalence of smoking cessation treatment in urology clinics? How concordant with guideline recommendations (behavioral counseling and pharmacotherapy) is this care? What is the variation in smoking cessation care delivered by urologists and what are factors associated with it? What are common barriers at the physician-level and how can they be overcome? In order to address these questions, our collaborative group is exploring factors at the population, health system, and individual provider-levels to better understand how urologists currently treat smokers and how we can better do so. Greater research funding and focus on developing strategies and interventions to overcome barriers and leverage facilitators to smoking cessation treatment will be important to improve urological care and help our patients quit smoking.

\section{Acknowledgments}

Funding: None.

\section{Footnote}

Provenance and Peer Review: This article was commissioned by the editorial office, Translational Andrology and Urology. The article did not undergo external peer review.

Conflicts of Interest: All authors have completed the ICMJE uniform disclosure form (available at http:// dx.doi.org/10.21037/tau-20-1204). MAB reports that he is supported by the New York State, Department of Health, Empire Clinical Research Investigator Program. He is principal investigator on grant UL1TR002489 "Characterization of Bladder Carcinogen Urinary Biomarkers in Young Adult Electronic Cigarette Users". $\mathrm{He}$ is principal investigator on University of North Carolina, Lineberger Comprehensive Cancer Center grant "Evaluating the carcinogenic effects of electronic cigarettes on the urothelium". The other authors have no conflicts of interest to declare.

Ethical Statement: The authors are accountable for all aspects of the work in ensuring that questions related to the accuracy or integrity of any part of the work are appropriately investigated and resolved.

Open Access Statement: This is an Open Access article distributed in accordance with the Creative Commons Attribution-NonCommercial-NoDerivs 4.0 International License (CC BY-NC-ND 4.0), which permits the noncommercial replication and distribution of the article with the strict proviso that no changes or edits are made and the original work is properly cited (including links to both the formal publication through the relevant DOI and the license). See: https://creativecommons.org/licenses/by-nc-nd/4.0/.

\section{References}

1. Danaei G, Ding EL, Mozaffarian D, et al. The preventable causes of death in the United States: comparative risk assessment of dietary, lifestyle, and metabolic risk factors. PLoS Med 2009;6:e1000058.

2. van Osch FH, Jochems SH, van Schooten FJ, et al. Quantified relations between exposure to tobacco smoking and bladder cancer risk: a meta-analysis of 89 observational studies. Int J Epidemiol 2016;45:857-70.

3. Hunt JD, van der Hel OL, McMillan GP, et al. Renal cell carcinoma in relation to cigarette smoking: meta-analysis of 24 studies. Int J Cancer 2005;114:101-8.

4. McVary KT, Carrier S, Wessells H, et al. Smoking and erectile dysfunction: evidence based analysis. J Urol 2001;166:1624-32.

5. Allen MS, Walter EE. Health-Related Lifestyle Factors and Sexual Dysfunction: A Meta-Analysis of PopulationBased Research. J Sex Med 2018;15:458-75.

6. Darcey E, Boyle T. Tobacco smoking and survival after a prostate cancer diagnosis: A systematic review and metaanalysis. Cancer Treat Rev 2018;70:30-40.

7. Parsons JK. Urological cancers and lifestyle: Nudging patients toward healthy behaviors. Urol Oncol 2019;37:357-8.

8. Cumberbatch MG, Rota M, Catto JWF, et al. The Role of Tobacco Smoke in Bladder and Kidney Carcinogenesis: A Comparison of Exposures and Meta-analysis of Incidence and Mortality Risks. Eur Urol 2016;70:458-66.

9. Bourke L, Bauld L, Bullen C, et al. E-cigarettes and Urologic Health: A Collaborative Review of Toxicology, Epidemiology, and Potential Risks. Eur Urol 2017;71:915-23.

10. Bjurlin MA, Goble SM, Hollowell CMP. Smoking cessation assistance for patients with bladder cancer: a national survey of American urologists. J Urol 2010;184:1901-6.

11. Bassett JC, Gore JL, Chi AC, et al. Impact of a bladder cancer diagnosis on smoking behavior. J Clin Oncol 2012;30:1871-8. 
12. Gild P, Vetterlein MW, Seiler R, et al. The association of cigarette smoking and pathological response to neoadjuvant platinum-based chemotherapy in patients undergoing treatment for urinary bladder cancer - A prospective European multicenter observational study of the EAU Young academic Urologists (YAU) urothelial carcinoma Working Group. Surg Oncol 2020;34:312-7.

13. Cacciamani GE, Ghodoussipour S, Mari A, et al. Association between Smoking Exposure, Neoadjuvant Chemotherapy Response and Survival Outcomes following Radical Cystectomy: Systematic Review and MetaAnalysis. J Urol 2020;204:649-60.

14. Rink M, Zabor EC, Furberg H, et al. Impact of smoking and smoking cessation on outcomes in bladder cancer patients treated with radical cystectomy. Eur Urol 2013;64:456-64.

15. Crivelli JJ, Xylinas E, Kluth LA, et al. Effect of smoking on outcomes of urothelial carcinoma: a systematic review of the literature. Eur Urol 2014;65:742-54.

16. Adams JM. Smoking Cessation-Progress, Barriers, and New Opportunities: The Surgeon General's Report on Smoking Cessation. JAMA 2020;323:2470-1.

17. CDC. Smoking Cessation: A report of the Surgeon General. 2020. Available online: https://www.cdc.gov/tobacco/data statistics/sgr/2020-smoking-cessation/index.html

18. Bassett JC, Gore JL, Kwan L, et al. Knowledge of the harms of tobacco use among patients with bladder cancer. Cancer 2014;120:3914-22.

19. Macleod LC, Dai JC, Holt SK, et al. Underuse and underreporting of smoking cessation for smokers with a new urologic cancer diagnosis. Urol Oncol 2015;33:504.e1-7.

20. Bjurlin MA, Cohn MR, Kim DY, et al. Brief smoking cessation intervention: a prospective trial in the urology setting. J Urol 2013;189:1843-9.

21. Stark T, Shoag JE, Nicolas J, et al. Ambulatory Bladder Cancer Care in the United States. Urology Practice 2019;6:165-73.

22. Gritz ER, Talluri R, Fokom Domgue J, et al. Smoking Behaviors in Survivors of Smoking-Related and Non-Smoking-Related Cancers. JAMA Netw Open 2020;3:e209072.

23. Chang SS. Re: Smoking Cessation: A Report of the Surgeon General. J Urol 2020;204:384.

24. Institute of Medicine (US) Committee on Quality of Health Care in America. Crossing the Quality Chasm: A New Health System for the 21st Century. Washington (DC): National Academies Press (US), 2001.

25. Tabak RG, Khoong EC, Chambers DA, et al. Bridging research and practice: models for dissemination and implementation research. Am J Prev Med 2012;43:337-50.

26. Smith AB, Brooke BS. How Implementation Science in Surgery is Done. JAMA Surg 2019;154:891-2.

27. Liang L, Abi Safi J, Gagliardi AR, et al. Number and type of guideline implementation tools varies by guideline, clinical condition, country of origin, and type of developer organization: content analysis of guidelines. Implement Sci 2017;12:136.

28. Henry RC, Ogle KS, Snellman LA. Preventive medicine: physician practices, beliefs, and perceived barriers for implementation. Fam Med 1987;19:110-3.

29. Reda AA, Kaper J, Fikrelter H, et al. Healthcare financing systems for increasing the use of tobacco dependence treatment. Cochrane Database Syst Rev 2009;(2):CD004305.

30. Rubenstein LV, Mittman BS, Yano EM, et al. From understanding health care provider behavior to improving health care: the QUERI framework for quality improvement. Quality Enhancement Research Initiative. Med Care 2000;38:1129-41.

31. Keith RE, Crosson JC, O'Malley AS, et al. Using the Consolidated Framework for Implementation Research (CFIR) to produce actionable findings: a rapid-cycle evaluation approach to improving implementation. Implement Sci 2017;12:15.

32. Atkins L, Francis J, Islam R, et al. A guide to using the Theoretical Domains Framework of behaviour change to investigate implementation problems. Implement Sci 2017;12:77.

33. Croyle RT, Morgan GD, Fiore MC. Addressing a Core Gap in Cancer Care - The NCI Moonshot Program to Help Oncology Patients Stop Smoking. N Engl J Med 2019;380:512-5.

34. Kirk MA, Haines ER, Rokoske FS, et al. A case study of a theory-based method for identifying and reporting core functions and forms of evidence-based interventions. Transl Behav Med 2019. [Epub ahead of print]. doi: 10.1093/tbm/ibz178.

35. Kirk MA, Hanson LC, Weinberger M, et al. Pilot Test of an Adapted Intervention to Improve Timeliness of Referrals to Hospice and Palliative Care for Eligible Home Health Patients. J Palliat Med 2019;22:1266-70.

Cite this article as: Matulewicz RS, Makarov DV, Sherman SE, Birken SA, Bjurlin MA. Urologist-led smoking cessation: a way forward through implementation science. Transl Androl Urol 2021;10(1):7-11. doi: 10.21037/tau-20-1204 